\title{
Co-infection with HPV types from the same species provides natural cross-protection from progression to cervical cancer
}

\author{
Rafal S Sobota ${ }^{1,2,6^{*}}$, Doreen Ramogola-Masire ${ }^{3,4,5}$, Scott M Williams ${ }^{2}$ and Nicola M Zetola ${ }^{3,4,5^{*}}$
}

\begin{abstract}
Background: The worldwide administration of bivalent and quadrivalent HPV vaccines has resulted in cross-protection against non-vaccine HPV types. Infection with multiple HPV types may offer similar cross-protection in the natural setting. We hypothesized that infections with two or more HPV types from the same species, and independently, infections with two or more HPV types from different species, associate with protection from high-grade lesions.

Findings: We recruited a cohort of 94 HIV, HPV-positive women from Botswana, with Grade 2 or higher cervical intraepithelial neoplasia. Infections with 2 or more HPV types from a single species associated with reduced lesion severity in univariate analysis $(\mathrm{OR}=0.41,95 \% \mathrm{Cl} 0.18-0.97, \mathrm{p}=0.042)$, when adjusted for the presence of HPV 16 or 18 types $(\mathrm{OR}=0.41,95 \% \mathrm{Cl} 0.17-1.00, \mathrm{p}=0.049)$, or all high-risk HPV type infections $(\mathrm{OR}=0.38,95 \% \mathrm{Cl} 0.16-0.90, \mathrm{p}=0.028)$. Infections with 2 or more HPV types from different species did not associate ( $\mathrm{OR}=0.68,95 \% \mathrm{Cl} 0.25-1.81, \mathrm{p}=0.435$ ).

Conclusions: Our findings show that co-infections with genetically similar HPV types reduce the likelihood of progression to high-grade lesions in HIV positive women, an effect not observed in co-infections with taxonomically different HPV types. This observation is possibly caused by an immune cross-protection through a similar mechanism to that observed after HPV vaccination.
\end{abstract}

Keywords: HPV, Taxonomy, Cervical cancer, Coinfection, Immune cross-protection, Vaccine

\section{Background}

Cervical cancer is the third most common cancer in women worldwide and the number one cause of cancerrelated mortality in Sub-Saharan Africa [1]. Infection with an oncogenic human papillomavirus (HPV) type is necessary, but not sufficient, for progression to cervical cancer [2]. Most HPV types do not cause cancer, and position on the viral phylogeny affects disease risk [3,4]. HPV phylogeny is based on genetic distance between isolates, with the genus serving as the broadest taxon. Within a genus, similar viruses are classified into species, and viruses within a species are subdivided into types [5,6]. Only the alpha genus of HPV is associated with cervical cancer [7]. The carcinogenic HPV types fall

\footnotetext{
*Correspondence: rafal.s.sobota@vanderbilt.edu; nzetola@gmail.com ${ }^{1}$ Center for Human Genetics Research, Department of Molecular Physiology and Biophysics, Vanderbilt University Medical Center, Nashville, TN, USA ${ }^{3}$ Division of Infectious Disease, University of Pennsylvania, Philadelphia, PA, USA

Full list of author information is available at the end of the article
}

into 5 species, $\alpha 5, \alpha 6, \alpha 7, \alpha 9$ and $\alpha 11$ [3] and are termed high-risk species. Two HPV types, 16 and 18, are responsible for approximately $70 \%$ of cervical cancer cases worldwide; with the majority of the remainder ascribed to 11 other types [8].

Vaccination against HPV was a major advance in cervical cancer prevention, offering protection against a few disease causing types. Currently, 2 prophylactic vaccines are approved for human use. The tetravalent vaccine covers four HPV types, offering protection against cervical cancer (types 16 and 18) as well as genital warts (types 6 and 11). The bivalent vaccine covers types 16 and 18 , targeting only cervical cancer $[5,6,9]$. Understanding the effects of HPV co-infections is essential in determining the long term effects of the vaccines. Availability of such results can better inform the projections on the effect of broad vaccine implementation, and the subsequent prevalence of cervical cancer. Available data suggest that both vaccines have a variable level of crossprotection against types closely related HPV types [10]. 
It is hypothesized that this protection can be attributed to the genetic similarity between the vaccine types and those against which cross-protection is afforded, as the types for which cross-protection was observed are in the same species as HPV 16 and 18.

In this study we determined whether taxonomic relationships of co-infecting HPV types associated with progression to advanced cervical lesions (CIN3+). We tested two independent hypotheses: 1) infections with two or more HPV types from the same species associate with progression to high-grade lesions; or 2) infections with two or more HPV types from different species associate with progression to high-grade lesions.

\section{Findings}

We recruited HIV-positive women living in Botswana who were diagnosed with a Grade 2 or higher cervical intraepithelial neoplasia (CIN2 or above) on colposcopy. All patients provided informed consent.

Specimen collection and processing have been previously described [11]. Briefly, Pap smear swabs were lysed in $500 \mu \mathrm{L}$ of lysis buffer (Roche MagNA Pure LC DNA Isolation Kit) for 30 minutes at room temperature. DNA was extracted, amplified and analyzed following the manufacturer's specifications (Roche Linear Array ${ }^{\circ} \mathrm{HPV}$ Genotyping test). This method can detect 13 high-risk HPV genotypes (genotypes 16, 18, 31, 33, 35, 39, 45, 51, $52,56,58,59$, and 68 ) and 24 low-risk HPV genotypes (genotypes 6, 11, 26, 40, 42, 53, 54, 55, 61, 62, 64, 66, 67, 69, 70, 71, 72, 73, 81, 82, 83, 84, IS39, and CP6108).

HPV types were assigned to species using conventional taxonomic criteria [5,6]. Briefly, using the L1 ORF of papillomaviruses, viruses with $<60 \%$ sequence homology in this region are considered to be in a different genus. Sequence homology between 60 and $70 \%$ constitutes different species, while different HPV types within a species have between 70 and $89 \%$ nucleotide identity $[5,6]$. Only HPV types in the alpha-papillomavirus genus were considered in our study.

Univariate logistic regression was used to determine association between CIN3 lesions (as opposed to CIN2) and three categories of HPV co-infection, separately. The HPV co-infection categories were: 1) two or more HPV types from two or more species, 2) two or more HPV types from a single species, 3) two or more HPV types from a single high-risk species $(\alpha 5, \alpha 6, \alpha 7, \alpha 9$ or $\alpha 11$ [3]).

We also tested the association between CIN3 lesions and variables previously associated with CIN progression; namely: baseline CD4 count, patient age at the time of screening, patient age at the time of first intercourse, and lifetime number of sexual partners [12]. Univariate logistic regression was performed between presence of CIN3 and each variable. Univariate analyses were used to separately model the three categories of patient HPV as the dependent variable and patient CD4 counts either at presentation or the last available $\mathrm{CD} 4$ value as the independent variables.

Multivariate logistic regression models of CIN3 were tested, separately using the $3 \mathrm{HPV}$ co-infection categories above, along with other variables statistically significant in univariate analyses. Presence of HPV 16 or 18, or the presence of a high-risk HPV of any type was also included in the model.

\section{Study population}

94 women with CIN2, CIN3, and squamous cell carcinoma made up the final study population. 41 of the patients had CIN2, 52 had CIN3, and one had squamous cell carcinoma (SCC). The squamous cell carcinoma patient was categorized as CIN3 in the logistic regression analyses (ordinal analyses with a separate SCC category yielded comparable results, Additional file 1: Table S1). The mean age of study participants was 36.78 and the median number of HPV type co-infections per patient was 3 (Table 1).

The classification of HPV types into species is presented in Additional file 1: Table S2 [13]. 79 patients were infected with multiple HPV types. 72 patients had two or more HPV types from different species, 53 patients had two or more HPV types from a single species, and 40 patients had two or more HPV types from a single high-risk species. We found co-infections with only single HPV types of multiple species in 26 patients. Seven patients were infected with multiple HPV types from one species exclusively, all of which were high-risk species. 46 patients had infections with both HPV types in multiple species and multiple HPV types in the same species. 90 patients were infected with a high-risk HPV type, while 50 patients had an infection with either HPV 16 or 18 .

\section{Univariate analyses}

Infection with two or more HPV types from a single species protected against progression to higher risk lesions ( $\mathrm{OR}=0.41,95 \%$ CI 0.18-0.97, $\mathrm{p}=0.042$; Table 2). Infection with two or more HPV from a single high-risk

Table 1 Summary statistics of study population

\begin{tabular}{lll}
\hline Variable & Mean (st dev) & Median (IQR) \\
\hline Age & $36.78(5.90)$ & $36(33-39)$ \\
HPV infections per person & $3.93(2.62)$ & $3(2-5)$ \\
Age at first intercourse* & $18.13(2.32)$ & $18(16-20)$ \\
Number of lifetime sexual partners* & $5.86(5.26)$ & $4(3-6)$ \\
CD4 count during first visit** & $254.85(207.29)$ & $200(109-365)$ \\
CD4 count during last visit & $452.43(208.12)$ & $426(285-578)$ \\
\hline
\end{tabular}

Data available for 63 patients* and for 61 patients**. 
Table 2 Univariate and multivariate logistic regression results modeling CIN3 presence/absence or HPV co-infections

\begin{tabular}{|c|c|c|c|c|}
\hline Outcome & Covariate(s) & Odds ratio & $95 \% \mathrm{Cl}$ & $\mathrm{p}$ value \\
\hline Presence of CIN3 & Multiple types single species & 0.4145 & $0.177-0.970$ & 0.042 \\
\hline Presence of CIN3 & Multiple types different species & 0.6753 & $0.252-1.808$ & 0.435 \\
\hline Presence of CIN3 & Multiple types single high-risk species & 0.3696 & $0.159-0860$ & 0.021 \\
\hline Presence of CIN3 & HPV 16 or 18 & 0.8127 & $0.358-1.843$ & 0.620 \\
\hline Presence of CIN3 & Age & 1.0204 & $0.951-1.095$ & 0.576 \\
\hline Presence of CIN3 & Sexual Partners* & 0.9997 & $0.909-1.099$ & 0.994 \\
\hline Presence of CIN3 & Age at First Sex* & 1.0722 & $0.861-1.336$ & 0.534 \\
\hline Presence of CIN3 & CD4 count at presentation $\uparrow$ & 1.0014 & $0.998-1.004$ & 0.297 \\
\hline Presence of CIN3 & Last available CD4 count & 1.0003 & $0.998-1.002$ & 0.746 \\
\hline Presence of CIN3 & Multiple types from single species; high-risk HPV尹 & 0.3783 & $0.159-0.899$ & 0.028 \\
\hline Presence of CIN3 & Multiple types from single species; HPV 16 or 18 & 0.4138 & $0.172-0.996$ & 0.049 \\
\hline Presence of CIN3 & Multiple types from different species; high-risk HPVキ & 0.6061 & $0.225-1.632$ & 0.322 \\
\hline Presence of CIN3 & Multiple types from different species; HPV 16 or 18 & 0.6980 & $0.256-1.900$ & 0.482 \\
\hline Presence of CIN3 & Multiple types from single high-risk species; high-risk HPVキ & 0.4158 & $0.177-0.975$ & 0.044 \\
\hline Presence of CIN3 & Multiple types from single high-risk species; HPV 16 or 18 & 0.3661 & $0.155-0.847$ & 0.024 \\
\hline 2+ HPV infections & CD4 count at presentation & 0.9978 & $0.995-1.001$ & 0.168 \\
\hline $2+\mathrm{HPV}$ infections & Last available CD4 count & 0.9980 & $0.995-1.001$ & 0.130 \\
\hline Multiple types single species & CD4 count at presentation & 1.0001 & $0.998-1.002$ & 0.963 \\
\hline Multiple types single species & Last available CD4 count & 0.9995 & $0.998-1.001$ & 0.618 \\
\hline Multiple types single high-risk species & CD4 count at presentation & 1.0006 & $0.998-1.003$ & 0.623 \\
\hline Multiple types single high-risk species & Last available CD4 count & 1.0008 & $0.999-1.003$ & 0.413 \\
\hline Multiple types different species & CD4 count at presentation & 0.9980 & $0.995-1.001$ & 0.187 \\
\hline Multiple types different species & Last available CD4 count & 0.9981 & $0.996-1.000$ & 0.106 \\
\hline
\end{tabular}

Data available for 63 patients* and for 61 patientst. 90 patients had high risk HPV typesf.

species was also protective $(\mathrm{OR}=0.37,95 \%$ CI $0.16-0.86$, $\mathrm{p}=0.021$ ). Infection with two or more HPV types from different species did not associate with progression (OR $=0.68,95 \%$ CI 0.25-1.81, $\mathrm{p}=0.435$ ).

Univariate regression of CIN3 with a patient's CD4 count at first presentation, last available CD4 count, patient's age, number of lifetime sexual partners, and most of the individual high-risk HPV types were not statistically significant (Table 2, Additional file 1: Table S3). Patients with HPV types 39 were always diagnosed with CIN3, and those with HPV 26, 40, or 73 always had CIN2 (Additional file 1: Table S3). HPV 51 also associated with CIN3 (OR $=0.16,95 \%$ CI $0.03-0.81, \mathrm{p}=0.027$ ) (Additional file 1: Table S3). In our sample, infection with either HPV 16 or 18 was not associated with progression to CIN3 $(\mathrm{p}=0.62)$. Initial and last available CD4 counts were not associated with the presence of any of the HPV co-infection categories (Table 2).

\section{Multivariate analyses}

In multivariate analysis, the association of CIN3 with two or more HPV type infections from multiple species was not significant when adjusting for high-risk HPV type infections $(\mathrm{OR}=0.61,95 \% \mathrm{CI} 0.22-1.63, \mathrm{p}=0.322$; Table 2). Association of CIN3 with two or more HPV type infections from a single species was significant when adjusting for high-risk HPV type infections (single species co-infection OR $=0.38,95 \%$ CI $0.16-0.90, \mathrm{p}=$ 0.028; Table 2). Infections with two or more HPV types from a single high-risk species were significant in multivariate analyses adjusted for all high-risk HPV (OR = 0.42, 95\% CI 0.18-0.97, $\mathrm{p}=0.044$; Table 2).

\section{Discussion}

Our results indicate that the presence of infections with two or more HPV types from the same species protects from progression to high-grade CIN. While our data did not longitudinally ascertain the sequence of infection events, it is likely that some portion of our patients were infected with a low-risk HPV type prior to the acquisition of a high-risk type from the same species. This sequence of events could boost the immune system against the high-risk types and provide cross-protection via a mechanism similar to that afforded for HPV 31 and 45 infections when using vaccines [14]. 
Cross-reactive immune response based on taxonomic placement has been previously considered among HPV vaccines [15]. Immune responses to natural infection have been shown to be weaker than those elicited by the vaccines [14]. However, the persistence of HPV infections coupled with the immunodominant nature of the response, generating antibodies to only a subset of antigens, makes natural cross-protection an important factor to consider [16]. Cross-reactivity could offer a degree of protection capable of preventing or delaying carcinogenesis to only a subset of similar type infections (same species) but not strong enough to clear the infection. This mechanism could account for the effects observed in our study. This conclusion is further supported by the result that coinfections with HPV types from different species did not associate with protection for progressing to CIN3, while univariate analyses of same species infections in only the five high-risk species provided stronger associations and effect sizes than same species infections in general (Table 2, Additional file 1: Table S1). Hence sequence similarity among co-infections increases protection.

HIV and resultant low CD4 counts have been previously associated with increased risk of single and multiple HPV infections [17]. However, in our study, HIV-associated immunosuppression was not predictive of multiple HPV infections (Table 2). We observed no effect of CD4 counts on the likelihood of acquisition of co-infections with multiple HPV species, regardless of category (Table 2). This leads us to suggest that our findings are applicable beyond the niche of HIV + patients.

Our data, observed in an unvaccinated, natural setting, indicate that protection from any high-risk species type is likely to confer some degree of protection against all types in that species, supporting the argument that vaccines would do better if all high-risk species were covered. Therefore, widespread use of HPV vaccines can indirectly affect the prevalence of some non-vaccine types. Since the bivalent vaccine covers species $\alpha 7$ and $\alpha 9$, and the quadrivalent adds protection to $\alpha 10$, the burden of HPV carcinogenesis in a fully vaccinated population might shift to the high-risk species that are not currently covered by either vaccine; namely $\alpha 5, \alpha 6$, and $\alpha 11$. Adding virus-like particles from at least one HPV type in each of these species should therefore be a priority for future vaccine development.

This study was reviewed and approved by the Institutional Review Boards of the University of Pennsylvania and the Botswana Ministry of Health.

\section{Additional files}

Additional file 1: Table S1. Univariate and multivariate ordinal

regression modeling lesion severity, $\mathrm{CIN} 2=0, \mathrm{CIN} 3=1$, and $\mathrm{SCC}=2$, with

co-infection categories and relevant covariates. Table S2. Assignment of
HPV types found in this study into species of the alpha-papillomavirus genus. Table S3. Univariate logistic regression results of progression to CIN 3 versus individual HPV types.

\section{Competing interests}

The authors declare that they have no competing interests.

\section{Authors' contributions}

NZ and RS had full access to all the data in the study and take responsibility for the integrity of the data and the accuracy of the data analysis. Study concept and design: RS, DR, SW and NZ. Acquisition of data: NZ and DR. Analysis and interpretation of data: RS, DR, SW and NZ. Drafting of manuscript: RS. Critical revision of the manuscript for important intellectual content: RS, DR, SW and NZ. Statistical analysis: RS, SW and NZ. Obtaining funding: NZ. Administrative, technical, or material support: DR and NM. Study supervision: SW and NZ. All authors read and approved the final manuscript.

\section{Acknowledgements}

RSS was supported by Public Health Service award T32 GM07347 from the National Institute of General Medical Studies for the Vanderbilt Medical-Scientist Training Program. SMW and RSS were partially supported by NIH grant P20 GM103534. NMZ was supported in part by NIH grants R01Al097045 and P30Al45008 (Penn Center for AIDS Research). The funders had no role in study design, data collection and analysis, decision to publish, or preparation of the manuscript.

\section{Author details}

${ }^{1}$ Center for Human Genetics Research, Department of Molecular Physiology and Biophysics, Vanderbilt University Medical Center, Nashville, TN, USA. ${ }^{2}$ Department of Genetics, Geisel School of Medicine, Dartmouth College, Hanover, NH, USA. ${ }^{3}$ Division of Infectious Disease, University of Pennsylvania, Philadelphia, PA, USA. ${ }^{4}$ Botswana-University of Pennsylvania Partnership, 214 Independence Ave, Gaborone, Botswana. ${ }^{5}$ Department of Medicine, University of Botswana, Gaborone, Botswana. ${ }^{6}$ Center for Human Genetics Research, Vanderbilt University, 519 Light Hall, 2215 Garland AveNashville, TN 37232, USA.

Received: 4 April 2014 Accepted: 22 July 2014

Published: 12 August 2014

\section{References}

1. Ferlay J, Shin HR, Bray F, Forman D, Mathers C, Parkin DM: Estimates of worldwide burden of cancer in 2008: GLOBOCAN 2008. Int J Canc J Int Canc 2010, 127(12):2893-2917.

2. Walboomers JM, Jacobs MV, Manos MM, Bosch FX, Kummer JA, Shah KV Snijders PJ, Peto J, Meijer CJ, Munoz N: Human papillomavirus is a necessary cause of invasive cervical cancer worldwide. J Pathol 1999, 189(1):12-19.

3. Schiffman M, Rodriguez AC, Chen Z, Wacholder S, Herrero R, Hildesheim A, Desalle R, Befano B, Yu K, Safaeian M: A population-based prospective study of carcinogenic human papillomavirus variant lineages, viral persistence, and cervical neoplasia. Cancer Res 2010, 70(8):3159-3169.

4. Burk RD, Chen Z, Van Doorslaer K: Human papillomaviruses: genetic basis of carcinogenicity. Publ Health Genomics 2009, 12(5-6):281-290.

5. de Villiers EM, Fauquet C, Broker TR, Bernard HU, zur Hausen $\mathrm{H}$ : Classification of papillomaviruses. Virology 2004, 324(1):17-27.

6. Bernard HU, Burk RD, Chen Z, van Doorslaer K, zur Hausen H, de Villiers EM: Classification of papillomaviruses (PVs) based on 189 PV types and proposal of taxonomic amendments. Virology 2010, 401(1):70-79.

7. Guindon S, Gascuel O: A simple, fast, and accurate algorithm to estimate large phylogenies by maximum likelihood. Syst Biol 2003, 52:696-704.

8. Doorslaer KV: Evolution of the Papillomaviridae. Virology 2013, 445:11-20.

9. Schiffman M, Clifford G, Buonaguro FM: Classification of weakly carcinogenic human papillomavirus types: addressing the limits of epidemiology at the borderline. Infect Agents Canc 2009, 4:8.

10. Clifford G, Franceschi S, Diaz M, Munoz N, Villa LL: Chapter 3: HPV type distribution in women with and without cervical neoplastic diseases. Vaccine 2006, 24(Suppl 3):26-34.

11. Barr E, Tamms G: Quadrivalent human papillomavirus vaccine. Clin Infect Dis: Offic Publ Infect Dis Soc Am 2007, 45(5):607-609. 
12. Jaquet A, Horo A, Ekouevi DK, Toure B, Coffie PA, Effi B, Lenaud S, Messou E, Minga A, Sasco AJ: Risk factors for cervical intraepithelial neoplasia in HIV-infected women on antiretroviral treatment in Côte d'Ivoire. West Africa PLoS One 2014, 9(3):e90625.

13. Keam SJ, Harper DM: Human papillomavirus types 16 and 18 vaccine (recombinant, AS04 adjuvanted, adsorbed) [Cervarix]. Drugs 2008, 68(3):359-372.

14. Mariani L, Venuti A: HPV vaccine: an overview of immune response, clinical protection, and new approaches for the future. J Transl Med 2010, 8:105.

15. Malagon T, Drolet M, Boily MC, Franco EL, Jit M, Brisson J, Brisson M: Cross-protective efficacy of two human papillomavirus vaccines: a systematic review and meta-analysis. Lancet Infect Dis 2012, 12(10):781-789.

16. Siddiqui MA, Perry CM: Human papillomavirus quadrivalent (types 6, $11,16,18)$ recombinant vaccine (Gardasil). Drugs 2006, 66(9):1263-1271. discussion 1272-1263.

17. Camargo M, Soto-De Leon SC, Munoz M, Sanchez R, Peňa-Herrera D, Pineda-Peňa AC, Sussmann O, Paez C, Perez-Prados A, Patarroyo ME: Human papillomavirus detection in women with and without human immunodeficiency virus infection in Colombia. BMC Cancer 2014, 14(1):451.

doi:10.1186/1750-9378-9-26

Cite this article as: Sobota et al: Co-infection with HPV types from the same species provides natural cross-protection from progression to cervical cancer. Infectious Agents and Cancer 2014 9:26.

\section{Submit your next manuscript to BioMed Central and take full advantage of:}

- Convenient online submission

- Thorough peer review

- No space constraints or color figure charges

- Immediate publication on acceptance

- Inclusion in PubMed, CAS, Scopus and Google Scholar

- Research which is freely available for redistribution 